\title{
Complutum
}

ISSN: 1131-6993

\section{Prácticas socioeconómicas de la fauna doméstica en la Edad del Hierro en el Valle} Medio del Tajo

\author{
Verónica Estaca-Gómez
}

Recibido: 16 de diciembre de 2016 / Aceptado: 22 de noviembre de 2018

Resumen. El estudio de las sociedades prehistóricas puede tratarse desde diferentes y variados ámbitos de conocimiento. A través de la cultura material podemos saber aspectos sociales, simbólicos, económicos o religiosos; en definitiva, las características principales de una sociedad. Mediante otras técnicas también podemos conocer su cronología o las condiciones paleoambientales en las que vivieron. En este trabajo abordamos algunos aspectos socioeconómicos de las sociedades asentadas en el Valle Medio del Tajo durante la Edad del Hierro, a través del estudio zooarqueológico de siete yacimientos inéditos.

Palabras clave: Edad del Hierro; Valle Medio del Tajo; Ganadería; Zooarqueología

[en] Socioeconomic practices of the domestic fauna from the Iron Age in the Middle Valley of Tajo

\begin{abstract}
Prehistoric societies may be studied from a variety of perspectives and areas. Material culture can be indicative of social, symbolic, economic or religious aspects, among others, embedding the main elements in a society. Different techniques inform about chronology or the palaeoenvironmental conditions of the time. This article discusses some socioeconomic activities of the societies settled in the Tajo middle basin during the Iron Age, through the zooarchaeological study of seven unpublished sites.
\end{abstract}

Keywords: Iron Age; Tajo middle basin; cattle herding; zooarchaeology

Sumario: 1. Introducción. 2. Localización de los yacimientos. 3. Materiales y métodos. 4. Resultados. 4.1. Patrones de representación taxonómica. 4.2. Patrones de mortandad. 4.3. Patrones de representación esquelética. 5. Discusión y puesta en valor de los datos en su contexto. 6. Conclusiones.

Cómo citar: Estaca-Gómez, V. (2018): Prácticas socioeconómicas de la fauna doméstica en la Edad del Hierro en el Valle Medio del Tajo. Complutum, 29(2): 387-406.

\section{Introducción}

Durante la última década se han ido desarrollando algunos trabajos que han permitido paliar la escasa información que existía para la Edad del Hierro en el Valle Medio del Tajo (Morín, et al., 2005; Dávila 2007; Morín y Urbina 2012; Torres 2013; Baquedano 2014; Azcárraga 2015). Los trabajos monográficos indicados nos han permitido tener una imagen general de las sociedades que vivieron durante la Edad del Hierro en el centro de la Península Ibérica.

A pesar de todos estos trabajos, la información disponible sobre las estrategias económicas, en lo que se refiere al aprovechamiento y explotación de los recursos animales, es muy limitada (Yravedra y Estaca 2014).

\footnotetext{
Departamento de Prehistoria, Historia Antigua y Arqueología. Universidad Complutense de Madrid. Avda. Profesor Aranguren $\mathrm{S} / \mathrm{N}, 28040$.- Madrid vestacag@hotmail.com
} 
Aun así se pueden destacar los trabajos de Morales (1980), Miguel (1985), Chaves et al. (1991), Cerdeño et al. (1992), Miguel y Morales (1994), Liesau (1998a y b), Urbina et al. (2005), Yravedra (2007a y b), Consuegra et al. (2007) y López y Morales (2012). Con esas referencias se articuló un discurso en el que se planteaba que estas poblaciones consumían ovicápridos como animal predominante, seguido de bóvidos y suidos. Sin embargo, estos datos no eran suficientes para mostrar los modos de vida económicos de esta sociedad.

En este trabajo se presentan los datos zooarqueológicos inéditos de siete yacimientos: La Guirnalda de Quer en la provincia de Guadalajara; La Gavia III, Humanejos, La Cuesta y Torrejón de Velasco pertenecientes a la Comunidad de Madrid y, por último, en la provincia de Toledo Entreviñas I y Cerrocuquillo. A partir de los datos obtenidos en estos sitios y la contextualización regional mostraremos al- gunas reflexiones sobe las prácticas ganaderas y/o socioeconómicas de las sociedades de la Edad del Hierro en el centro de la Península Ibérica.

\section{Localización de los yacimientos}

Todos los yacimientos se enmarcan en la cuenca media del Tajo, distribuidos en el ámbito territorial comprendido por el suroeste de Guadalajara, sureste de la Comunidad de Madrid y norte de Toledo (Fig. 1). De los conjuntos faunísticos analizados, La Guirnalda de Quer se sitúa en el área geográfica actual de la provincia de Guadalajara (Agustí 2007 y Agustí et al. 2012), los asentamientos de La Gavia III (Morín 2008a), Humanejos (Flores 2011), La Cuesta (Flores y Sanabria 2012 y 2014) y Torrejón de Velasco (Morín 2008b) están en la Comunidad de Madrid y los de Entreviñas I (Garrido 2012) y Cerrocuquillo (Baquedano et al. 2010 y Torija et al. 2010) en Toledo.

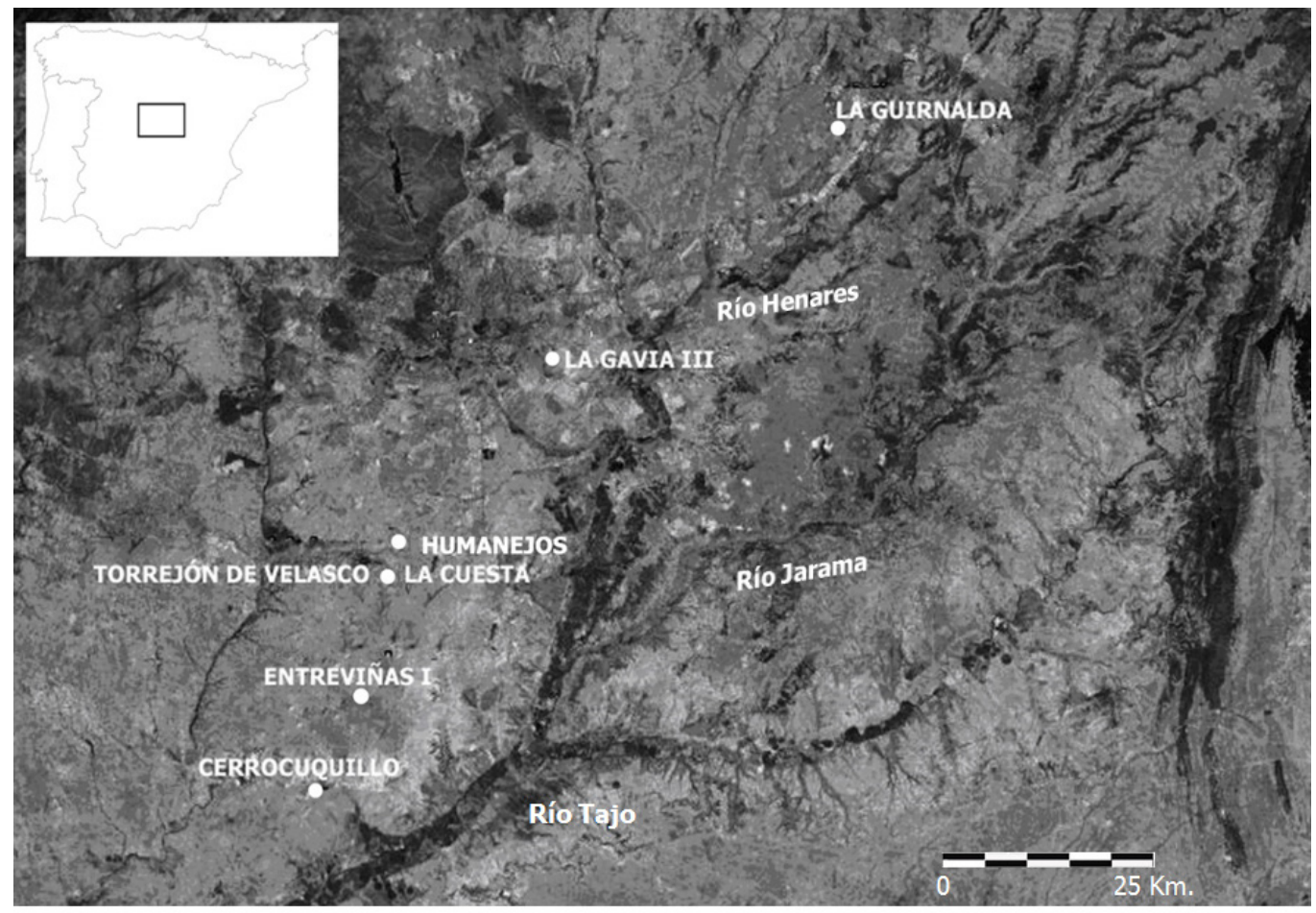

Figura 1. Situación geográfica de los yacimientos estudiados en este trabajo.

\section{Materiales y métodos}

El conjunto de fauna procesada para este trabajo es de 31.731 restos, de los cuales 10.826 pertenecen a la Primera Edad del Hierro y
20.016 a la segunda, además de 65 restos atribuibles a la Edad del Bronce Final y 824 al período de transición entre la Primera y la Segunda Edad del Hierro (Tabla 1). 


\begin{tabular}{|l|c|c|c|c|c|}
\hline Yacimientos & NR total & Bronce final & $\begin{array}{c}\text { Primera Edad } \\
\text { del Hierro }\end{array}$ & $\begin{array}{c}\text { Transición I/ } \\
\text { II EH }\end{array}$ & $\begin{array}{c}\text { Segunda Edad del } \\
\text { Hierro }\end{array}$ \\
\hline La Guirnalda & 3.926 & 65 & 2.299 & & 1.562 \\
\hline La Gavia III & 2.834 & & & & 2.834 \\
\hline Humanejos & 5.626 & & 113 & & 5.513 \\
\hline La Cuesta & 4.061 & & 669 & & 3.392 \\
\hline Entreviñas & 400 & & & & 400 \\
\hline Cerrocuquillo & 14.884 & & 7.745 & 824 & 6.315 \\
\hline Total & $\mathbf{3 1 . 7 3 1}$ & $\mathbf{6 5}$ & $\mathbf{1 0 . 8 2 6}$ & $\mathbf{8 2 4}$ & $\mathbf{2 0 . 0 1 6}$ \\
\hline
\end{tabular}

Tabla 1. Resumen de los datos obtenidos en el estudio de los yacimientos trabajados en la tesis en

NR total y por períodos.

En relación con la metodología empleada para este estudio lo primero a lo que vamos a referirnos es a la distribución de los perfiles taxonómicos. Para ello, se ha calculado el Número de Restos (NR) y el Mínimo Número de Individuos (MNI), también se han analizado los patrones de mortandad, y, por último, los perfiles esqueléticos.

Para la identificación taxonómica se ha seguido a Lavocat (1966), Pales y Lambert (1971), Schmid (1972), Martín y Blázquez (1983) y Hilson (1992). Para casos más específicos se ha seguido a Boessneck (1969), Payne (1985), Prummel y Frisch (1986) y Fernández (2001) para la diferenciación entre Ovis aries y Capra hircus, para el Bos taurus a Prummel (1988) y para diferenciar al jabalí del cerdo doméstico (Payne y Bull 1988). Junto a los trabajos de estos autores se ha manejado nuestra colección de referencia personal como medio de contraste. Así mismo para el cálculo del $N R$ se han incluido todos los restos, tanto identificables como no. El MNI se ha adscrito en función del resto anatómico más abundante diferenciando el lado izquierdo y derecho siguiendo a Brain (1969). En cuanto al MNI se ha optado por contabilizarlo de dos maneras, por un lado, considerando cada UE como unidades independientes entre sí. Y, por otro lado, con motivo de ofrecer el MNI de todo el conjunto, se ha contabilizado considerando los datos de todas las UU.EE. de cada sitio de manera conjunta.

Los patrones de edad se han podido determinar a partir de las piezas dentarias, observando el desgaste y la emergencia de los dientes definitivos respecto a los deciduales. Así como el grado de osificación de los huesos y la fusión de las epífisis. Los diferentes grupos de edad que se han tenido en cuenta son: infanti- les, juveniles y adultos. En relación con el cálculo de las edades se han seguido los trabajos de Pérez Ripoll (1988) y de Couturier (1962) para referirse a los ovicápridos, los de Mariezkurrena (1983), Brown y Chapman (1991 a y b) y Guadelli (1998) para los équidos.

Por último, se analizará la representación anatómica en la que se ha identificado la parte a la que pertenece el elemento óseo $y$, en los casos en los que esto no ha podido determinarse $\mathrm{y}$, por tanto, son huesos indeterminables, se los ha asignado a la categoría de axial, esponjosos (sí es tejido de alguna epífisis o algún hueso compacto) o diáfisis. De esta forma se pretende ver a qué elementos afecta más la fracturación. Además los diferentes huesos se han agrupado en distintas secciones: la porción craneal comprendida por los huesos del cráneo y la mandíbula, la porción axial integrada por vértebras, costillas, escápula y pelvis siguiendo las razones establecidas por Yravedra (2006) y el esqueleto apendicular que se subdivide en elementos superiores (húmero, fémur, tibia y radio-ulna) y el inferior formado por los metapodios y los huesos compactos.

\section{Resultados}

\subsection{Patrones de representación taxonómica}

Al analizar el NR se aprecia que, para la Primera Edad del Hierro, los animales domésticos están mejor representados que las especies silvestres (Tabla 2). Entre los animales domésticos la cabaña ganadera con mayor número de restos es la ovicaprina, destacando el caso del yacimiento de Cerrocuquillo, donde esta especie representa el 25\% del total de los restos estudiados, con una gran diferencia sobre 
el segundo taxón, los bóvidos con un 5\%, o en el caso de La Guirnalda donde los ovicápridos mantienen el porcentaje del $25 \%$ de los restos, pero los bóvidos están mejor representados en la muestra con un $19 \%$. De los yacimientos analizados sólo en La Cuesta los bóvidos su- peran ligeramente a los ovicápridos. Respecto al tercer taxón en importancia, en todos los sitios tratados predominan los cerdos, salvo la excepción de La Guirnalda donde destacan los équidos (Tabla 2).

\begin{tabular}{|c|c|c|c|c|c|}
\hline Taxón & La Guirnalda & Humanejos & La Cuesta & Cerrocuquillo & Total \\
\hline Bos taurus & 437 & 30 & 177 & 367 & 1.011 \\
\hline Equus caballus & 110 & 32 & 37 & 25 & 204 \\
\hline Equus asinus & 40 & & 1 & 5 & 46 \\
\hline Cervus elaphus & 100 & & 3 & 30 & 133 \\
\hline Ovis aries & 98 & 8 & 29 & 617 & 752 \\
\hline Capra hircus & 84 & & 16 & 37 & 137 \\
\hline Ovis/Capra & 404 & 27 & 100 & 1298 & 1.829 \\
\hline C. capreolus & 2 & & 2 & & 4 \\
\hline Sus scrofa & 4 & & & 3 & 7 \\
\hline Sus sp. & 123 & 10 & 52 & 187 & 372 \\
\hline Canis familiaris & 21 & & 10 & 32 & 63 \\
\hline Felis catus & 1 & & 1 & & 2 \\
\hline T. Grande & 572 & & 81 & 966 & 1.619 \\
\hline T. Media & 8 & & & 22 & 30 \\
\hline T. Pequeña & 244 & 6 & 116 & 3.578 & 3.944 \\
\hline Indet. & 51 & & 44 & 578 & 673 \\
\hline Total & 2.299 & 113 & 669 & 7.745 & 10.826 \\
\hline
\end{tabular}

Tabla 2. NR de los yacimientos pertenecientes a la Primera Edad del Hierro con relación a los taxones documentados.

En la Segunda Edad del Hierro destacan de nuevo los animales domésticos sobre los silvestres, cuya presencia es casi testimonial salvo en el caso del ciervo, gracias a la fragmentación de las astas. Además del ciervo se han documentado restos de otros animales como el corzo (Tabla 3).

Al igual que sucedía en el período anterior, entre los animales domésticos vuelven a predominar los ovicápridos con la excepción de
Entreviñas I, donde los restos más abundantes pertenecen a los bóvidos. Mención aparte tendría el caso de La Cuesta donde nos encontramos una relación pareja de predominancia entre ovicápridos y bóvidos. La segunda especie en importancia es la cabaña bovina, seguida de los suidos, con la excepción de La Gavia III donde ocupan el quinto lugar en importancia (Tabla 3). 


\begin{tabular}{|l|c|c|c|c|c|c|c|}
\hline Taxón & La Guirnalda & La Gavia III & Humanejos & La Cuesta & Entreviñas & Cerrocuquillo & Total \\
\hline Bos taurus & 276 & 323 & 939 & 668 & 59 & 476 & 2.741 \\
\hline Buey & 4 & & & & & & 4 \\
\hline Equus caballus & 61 & 123 & 122 & 68 & 7 & 153 & 534 \\
\hline Equus asinus & 12 & 31 & 11 & 55 & & 28 & 137 \\
\hline Cervus elaphus & 61 & 60 & 303 & 9 & 4 & 62 & 499 \\
\hline Ovis aries & 108 & 66 & 254 & 213 & 13 & 200 & 854 \\
\hline Capra hircus & 29 & 116 & 162 & 38 & & 21 & 366 \\
\hline Ovis/Capra & 263 & 261 & 1.194 & 410 & 21 & 1.029 & 3.178 \\
\hline C. capreolus & 4 & & 2 & 5 & & 1 & 12 \\
\hline Sus scrofa & 4 & 1 & 90 & 26 & & 9 & 130 \\
\hline Sus sp. & 125 & 114 & 702 & 443 & 24 & 398 & 1.806 \\
\hline Canis familiaris & 16 & 236 & 435 & 165 & 6 & 83 & 941 \\
\hline Felis catus & 1 & & & 1 & & & 2 \\
\hline T. Grande & 365 & 1.034 & 352 & 589 & 55 & 960 & 3.355 \\
\hline T. Media & 3 & 8 & 4 & 2 & 1 & & 28 \\
\hline T. Pequeña & 187 & 324 & 914 & 508 & 48 & 2.527 & 46 \\
\hline Indet. & 43 & 137 & 29 & 192 & 162 & 340 & 903 \\
\hline Total & $\mathbf{1 . 5 6 2}$ & $\mathbf{2 . 8 3 4}$ & $\mathbf{5 . 5 1 3}$ & $\mathbf{3 . 3 9 2}$ & $\mathbf{4 0 0}$ & $\mathbf{6 . 3 1 5}$ & $\mathbf{2 0 . 0 1 6}$ \\
\hline
\end{tabular}

Tabla 3. NR de los yacimientos pertenecientes a la Segunda Edad del Hierro con relación a los taxones documentados.

El análisis del MNI nos ha ofrecido para la Primera Edad del Hierro unos resultados parecidos a los de NR. De este modo son los ovicápridos el taxón con un mayor número de individuos, superando en la mayoría de los casos el $40 \%$ de los animales documentados en el yacimiento. Su predominancia oscila de unos lugares a otros de modo que en sitios como Humanejos alcanza el 33\% de los individuos y en otros como el Cerrocuquillo supera el 70\% de los individuos de la muestra. El segundo ta- xón en importancia es el grupo de los bóvidos, especialmente en La Guirnalda y Cerrocuqui1lo, mientras que en Humanejos y La Cuesta tiene una representación más moderada. El tercer grupo más importante es el de los suidos, con la excepción de La Guirnalda donde serían los équidos (Tabla 4).

Desde la perspectiva de analizar el MNI de cada taxón por UU.EE. se observa que los ovicápridos son el grupo más importante seguido de los bóvidos, suidos y équidos (Tabla 4).

\begin{tabular}{|l|c|c|c|c|c|c|c|c|c|c|}
\cline { 2 - 13 } \multicolumn{1}{c|}{} & \multicolumn{2}{c|}{ La Guirnalda } & \multicolumn{2}{c|}{ Humanejos } & \multicolumn{2}{c|}{ La Cuesta } & \multicolumn{2}{c|}{ Cerrocuquillo } & \multicolumn{2}{c|}{ Total } \\
\hline Taxón & General & UE & General & UE & General & UE & General & UE & General & UE \\
\hline Bos taurus & 15 & 52 & 1 & 2 & 6 & 33 & 7 & 52 & 29 & 139 \\
\hline Equus caballus & 3 & 21 & 1 & 1 & 2 & 7 & 1 & 17 & 7 & 46 \\
\hline Equus asinus & 5 & 13 & & & 1 & 1 & 1 & 5 & 7 & 19 \\
\hline Cervus elaphus & 2 & 16 & 1 & 2 & 2 & 3 & 1 & 14 & 6 & 35 \\
\hline Ovis aries & 9 & 25 & 1 & 1 & 5 & 11 & 20 & 45 & 35 & 82 \\
\hline Capra hircus & 10 & 17 & & & 4 & 5 & 6 & 13 & 20 & 35 \\
\hline Ovis/Capra & 11 & 51 & 1 & 6 & 5 & 25 & 16 & 96 & 33 & 178 \\
\hline C. capreolus & 2 & 2 & & & 1 & 2 & & & 3 & 4 \\
\hline Sus scrofa & 2 & 2 & & & & & 1 & 3 & 3 & 5 \\
\hline Sus sp. & 4 & 27 & 1 & 2 & 5 & 11 & 5 & 35 & 15 & 75 \\
\hline Canis familiaris & 5 & 8 & & & 1 & 3 & 1 & 10 & 7 & 21 \\
\hline Felis catus & 1 & 1 & & & 1 & 1 & & & 2 & 2 \\
\hline Total & $\mathbf{6 9}$ & $\mathbf{2 3 5}$ & $\mathbf{6}$ & $\mathbf{1 4}$ & $\mathbf{3 3}$ & $\mathbf{1 0 2}$ & $\mathbf{5 9}$ & $\mathbf{2 9 0}$ & $\mathbf{1 6 7}$ & $\mathbf{6 4 1}$ \\
\hline
\end{tabular}

Tabla 4. MNI general y por UU.EE. de los yacimientos pertenecientes a la Primera Edad del Hierro. 
Para el MNI de la Segunda Edad del Hierro vuelven a destacar los ovicápridos en todos los casos, con la excepción de Entreviñas I, donde el animal predomínate es el suido. El segundo grupo más importante en la mayoría de los sitios es el de los bóvidos, salvo en La Gavia III y Cerrocuquillo donde el MNI de équidos es muy parejo al de los bóvidos. De este modo los équidos suelen ser la tercera especie en importancia relegando a los suidos a la cuarta posición en todos los casos, salvo la ya mencionada excepción de Entreviñas I (Tabla 5).
Cuando se analiza el MNI por UU.EE. se observa el mismo panorama que en el MNI general, donde los dos taxones mejor representados son los ovicápridos y los bóvidos. Los únicos lugares en los que no se cumple esto es en Humanejos, debido a que el segundo taxón en importancia son los équidos, $\mathrm{y}$ en Entreviñas I en el que destacan los suidos. Con relación al tercer animal, encontramos una dualidad entre los équidos y los suidos, al presentar unas diferencias mínimas entre ellos (Tabla 5).

\begin{tabular}{|c|c|c|c|c|c|c|c|c|c|c|c|c|c|c|}
\hline \multirow[b]{2}{*}{ Taxón } & \multicolumn{2}{|c|}{ La Guirnalda } & \multicolumn{2}{|c|}{ La Gavia III } & \multicolumn{2}{|c|}{ Humanejos } & \multicolumn{2}{|c|}{ La Cuesta } & \multicolumn{2}{|c|}{ Entreviñas } & \multicolumn{2}{|c|}{ Cerrocuquillo } & \multicolumn{2}{|c|}{ Total } \\
\hline & General & UE & General & UE & General & UE & General & UE & General & UE & General & UE & General & UE \\
\hline $\begin{array}{l}\text { Bos } \\
\text { Taurus }\end{array}$ & 10 & 34 & 8 & 41 & & 30 & 12 & 58 & 2 & 8 & 10 & 39 & 42 & 210 \\
\hline Buey & 3 & 3 & & & & & & & & & & & 3 & 3 \\
\hline $\begin{array}{l}\text { Equus } \\
\text { caballus }\end{array}$ & 3 & 14 & 6 & 21 & & 32 & 4 & 16 & 1 & 4 & 7 & 22 & 21 & 109 \\
\hline $\begin{array}{l}\text { Equus } \\
\text { asinus }\end{array}$ & 3 & 5 & 3 & 11 & & & 2 & 8 & & & 3 & 9 & 11 & 33 \\
\hline $\begin{array}{l}\text { Cervus } \\
\text { elaphus }\end{array}$ & 2 & 14 & 2 & 12 & & 3 & 2 & 8 & 2 & 3 & 4 & 15 & 12 & 55 \\
\hline Ovis aries & 10 & 24 & 6 & 24 & & 27 & 17 & 25 & 1 & 5 & 11 & 29 & 45 & 134 \\
\hline $\begin{array}{l}\text { Capra } \\
\text { hircus }\end{array}$ & 5 & 11 & 3 & 4 & & & 2 & 12 & & & 3 & 7 & 13 & 34 \\
\hline $\begin{array}{l}\text { Ovis/ } \\
\text { Capra }\end{array}$ & 7 & 38 & 8 & 42 & & 27 & 11 & 55 & 2 & 11 & 11 & 58 & 39 & 231 \\
\hline $\begin{array}{l}\text { C. } \\
\text { capreolus }\end{array}$ & 1 & 2 & & & & & 1 & 3 & & & 1 & 1 & 3 & 6 \\
\hline Sus scrofa & 1 & 2 & 1 & 1 & & & 15 & 2 & & & 1 & 5 & 18 & 10 \\
\hline Sus sp. & 5 & 25 & 6 & 20 & & 10 & 2 & 45 & 6 & 11 & 6 & 25 & 25 & 136 \\
\hline $\begin{array}{l}\text { Canis } \\
\text { familiaris }\end{array}$ & 2 & 7 & 4 & 14 & & & 4 & 17 & 1 & 3 & 2 & 16 & 13 & 57 \\
\hline Felis catus & 1 & 1 & & & & & 1 & 1 & & & & & 2 & 2 \\
\hline Total & 53 & 180 & 47 & 190 & & 129 & 73 & 250 & 15 & 45 & 59 & 226 & 247 & 1.020 \\
\hline
\end{tabular}

Tabla 5. MNI general y por UU.EE. de los yacimientos pertenecientes a la Segunda Edad del Hierro.

\subsection{Patrones de mortandad}

$\mathrm{Al}$ analizar la mortandad en los yacimientos de la Primera Edad del Hierro se observa que los adultos son los individuos más frecuentes tanto en el MNI general como en el particular de cada UU. EE. (Fig. 2).

Hay que destacar que entre los individuos adultos que aparecen en los yacimientos hay un porcentaje de individuos adultos-seniles importante, esto se aprecia claramente en los casos de La Guirnalda y Cerrocuquillo. En La Guirnalda la frecuencia de individuos seniles oscila entre el 25 y el $40 \%$ y en Cerrocuquillo esta frecuencia va entre el 14 y el $17 \%$ de seniles entre los adultos, reflejando una menor importancia de este tipo de taxones. La presencia de individuos tan mayores nos indica que estos animales se rentabilizan durante toda su vida en el aprovechamiento de sus diferentes cualidades, como la explotación láctea en el caso de bovinos y ovicaprinos, o su fuerza de trabajo en tareas de tracción, laboreo agrícola o como medio de transporte en el caso de bovinos y équidos. 


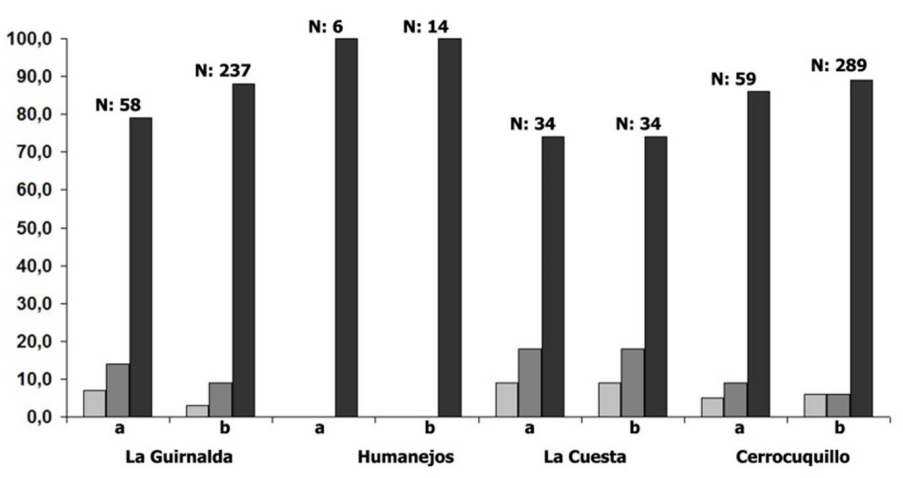

$\square$ Infantil $\square$ Juvenil $\square$ Adulto

\begin{tabular}{|c|c|c|c|c|c|c|c|c|}
\hline & \multicolumn{2}{|c|}{ La Guirnalda } & \multicolumn{2}{c|}{ Humanejos } & \multicolumn{2}{c|}{ La Cuesta } & \multicolumn{2}{c|}{ Cerrocuquillo } \\
\hline & $\mathrm{a}$ & $\mathrm{b}$ & $\mathrm{a}$ & $\mathrm{b}$ & $\mathrm{a}$ & $\mathrm{b}$ & $\mathrm{a}$ & $\mathrm{b}$ \\
\hline Infantil & 4 & 7 & & & 3 & & 3 & 16 \\
\hline Juvenil & 8 & 21 & & & 6 & & 5 & 16 \\
\hline Adulto & 46 & 209 & 6 & 14 & 25 & & 51 & 257 \\
\hline
\end{tabular}

Figura 2. MNI por edades tanto general como por UU. EE. para la Primera Edad del Hierro de todos los taxones. N: total de la muestra; a: general y b: UU.EE.

Los otros grupos de edad representados por infantiles y juveniles son relativamente escasos (Fig. 2). Esta baja representación de animales infantiles y juveniles determina que, en los diversos yacimientos estudiados las distintas cabañas animales no fueron aprovechadas exclusivamente para consumo cárnico. Esto nos sugiere que para este momento cronológico los animales eran llevados hasta el umbral más alto de edad para sacar de ellos todo el rendimiento económico posible a través del aprovechamiento de los llamados "productos secundarios", ya fuesen leche, lana, excrementos, fuerza de trabajo, etc., $y$, tras llegar a su cenit, se pasaba a su consumo cárnico. Del mismo modo la escasez de infantiles nos sugiere que la explotación cárnica de los animales no fue el objetivo prioritario de la producción ganadera.

Esto mismo se ve en la Segunda Edad del Hierro donde vuelve a ser el grupo de edad de los adultos el mejor representado, encontrando el mejor ejemplo en el yacimiento de Entreviñas I, donde todos los individuos documentados son adultos (Fig. 3).

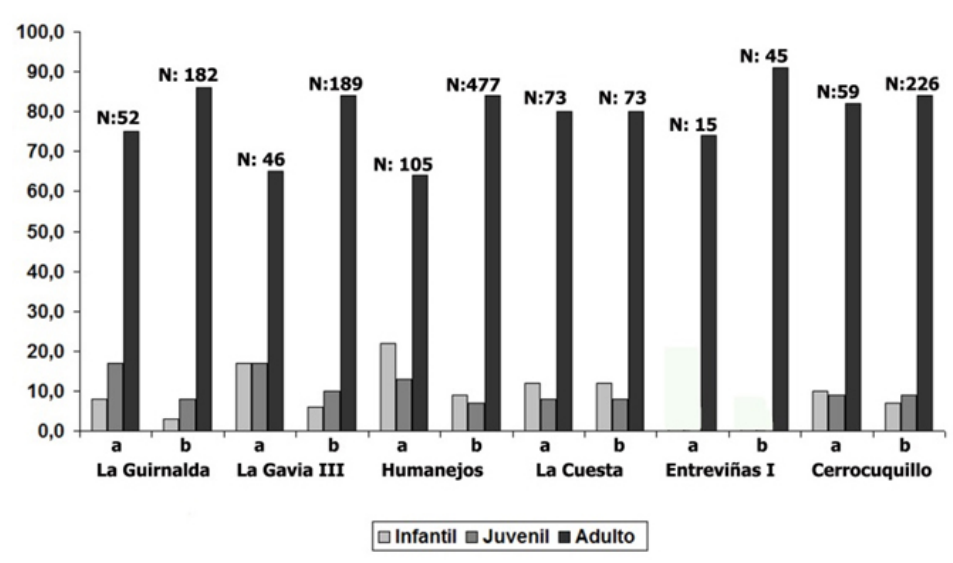

\begin{tabular}{|c|c|c|c|c|c|c|c|c|c|c|c|c|}
\hline & \multicolumn{2}{|c|}{ La Guirnalda } & \multicolumn{2}{c|}{ La Gavia II } & \multicolumn{2}{c|}{ Humanejos } & \multicolumn{2}{c|}{ La Cuesta } & \multicolumn{2}{|c|}{ Entreviñas I } & \multicolumn{2}{c|}{ Cerrocuquillo } \\
\hline & $\mathrm{a}$ & $\mathrm{b}$ & $\mathrm{a}$ & $\mathrm{b}$ & $\mathrm{a}$ & $\mathrm{b}$ & $\mathrm{a}$ & $\mathrm{b}$ & $\mathrm{a}$ & $\mathrm{b}$ & $\mathrm{a}$ & $\mathrm{b}$ \\
\hline Infantil & 4 & 6 & 8 & 11 & 23 & 45 & 9 & & & & 6 & 16 \\
\hline Juvenil & 9 & 15 & 8 & 19 & 14 & 33 & 6 & & & & 5 & 20 \\
\hline Adulto & 39 & 161 & 30 & 159 & 68 & 399 & 58 & & 11 & 45 & 48 & 190 \\
\hline
\end{tabular}

Figura 3. MNI por edades tanto general como por UU.EE. para la Segunda Edad del Hierro de todos los taxones. N: total de la muestra; a: general y b: UU.EE. 
Al analizar el grupo de los seniles se aprecia que en La Guirnalda alcanzan un porcentaje del $18 \%$ en bóvidos y ovicápridos, un valor que se dispara en los équidos debido a que los seniles representan el $50 \%$ de los individuos adultos, siendo un ejemplar de burro y el otro de caballo. En la Gavia III los porcentajes van desde los $14,3 \%$ en los équidos, al $20 \%$ en bóvidos y ovicápridos. Por último, los suidos tienen el mayor porcentaje de seniles con un $40 \%$. Este mismo dato se repite para los suidos en $\mathrm{Hu}-$ manejos, además de contar con un $20 \%$ entre los caballos, un $17,4 \%$ en los ovicápridos y un 15,38\% en los bóvidos. En Entreviñas I sólo se han podido obtener datos de seniles en el grupo de los suidos con un 33,3\%. Para finalizar, el yacimiento del Cerrocuquillo es, de entre todos los estudiados, el asentamiento que presenta los porcentajes más bajos de seniles, así sólo representan el $12,5 \%$ entre los bóvidos, el $11 \%$ en los caballos y el 9,5\% en los ovicápridos. Por tanto, en líneas generales, los rangos en los que se suele mover, a nivel porcentual, el valor para los seniles es de entre el $15 \%$ y el $30 \%$ de los adultos. Dentro de estos porcentajes los datos más altos los encontramos en los suidos, que van entre el $33 \%$ y el $40 \%$.

Según estos porcentajes se pueden hacer algunas valoraciones y reflexiones; la primera es que en relación a la Primera Edad del Hierro bajan sensiblemente las frecuencias de seniles, en segundo lugar destaca el porcentaje de seniles en una cabaña ganadera tradicionalmente asociada a la producción de carne. Nos referimos a los suidos con abundancia de seniles. Esto podría deberse a que los individuos representados son hembras de cerdos destinados a la cría, o bien al engorde y posterior consumo en jamones o salazones, o a que parte de los individuos representados en la categoría de Sus sp sean en realidad jabalíes cazados, y por tanto eso explicaría por qué son seniles.

En relación al resto de grupos de edad -infantiles y juveniles- son relativamente escasos, aunque ganan en importancia con respecto a la Primera Edad del Hierro. Si, como en el caso anterior, nos centramos en los neonatos, vemos que el número asciende. Mientras que en La Gavia III tenemos un individuo de bóvido y otro de ovicáprido, en Entreviñas I y Cerrocuquillo se han documentado un suido y un ovicáprido para cada yacimiento $\mathrm{y}$, por último, en Humanejos se localizó el mayor número de individuos prematuros, con 6 neonatos de suidos, de los cuales 4 son asignados a Sus sp. y 2 a Sus scrofa, junto con 4 perros.

\subsection{Patrones de representación esquelética}

Al analizar la representación anatómica en la Primera Edad del Hierro observamos que en casi todos los yacimientos se repiten unos patrones similares en los que suelen predominar las secciones craneales y apendiculares en casi todos los taxones, al contrario que la axial que suele ser la menos abundante (Tabla 6).

Así en La Guirnalda, suidos y cérvidos tienen un predominio de las secciones craneales $\mathrm{y}$ en los équidos y ovicápridos destacan los apendiculares. Por el contrario, en todos los taxones el esqueleto axial está peor representado, aunque en casos concretos como el caba1lo, la parte craneal es poco frecuente y en los suidos los apendiculares inferiores.

En La Cuesta el elemento mejor representado es la sección craneal para bóvidos, équidos y ovicápridos. Los axiales suelen estar mal representados salvo en los suidos. Es significativa también la escasez de elementos apendiculares superiores en la mayor parte de los taxones de este yacimiento.

En Humanejos, la muestra es tan exigua que no se puede dar ningún parámetro de comparación. En relación con Cerrocuquillo, se observa que en todos los taxones los elementos axiales son las partes peor representadas, a diferencia de la sección craneal en suidos y bóvidos y el apendicular en los ovicaprinos. 


\begin{tabular}{|l|c|c|c|c|c|c|c|c|}
\cline { 2 - 8 } \multicolumn{1}{c|}{} & \multicolumn{2}{c}{ La Guirnalda } & \multicolumn{2}{c}{ Lumanejos } & \multicolumn{2}{c|}{ Cerrocuquillo } \\
\cline { 2 - 8 } \multicolumn{1}{c|}{ Más } & Menos & Más & Menos & Más & Menos & Más & Menos \\
\hline Équido & Craneal & Axial & & & Craneal & $\begin{array}{c}\text { Axial/ Ap. } \\
\text { Superior }\end{array}$ & Craneal & Axial \\
\hline Ciervo & Ap. Inferior & Craneal & & & $\begin{array}{c}\text { Craneal/Ap. } \\
\text { Inferior }\end{array}$ & $\begin{array}{c}\text { Axial/ Ap. } \\
\text { Superior }\end{array}$ & & \\
\hline Ovicáprido & Ap. Superior & Axial & & & Craneal & Axial & Ap. Superior & Axial \\
\hline Suido & Craneal & Ap. Inferior & & & Axial & $\begin{array}{c}\text { Ap. Superior/ } \\
\text { Inferior }\end{array}$ & Craneal & Axial \\
\hline
\end{tabular}

Tabla 6. Representación de los yacimientos y los taxones más representativos en la muestra en relación a las secciones anatómicas más y menos abundantes.

Para la Segunda Edad del Hierro hemos observado una situación parecida a la descrita en la Primera Edad del Hierro, de modo que generalmente predominan los elementos craneales y los apendiculares y los axiales suelen estar mal representados (Tabla 7).

En La Guirnalda, los bóvidos, équidos y ovicápridos presentan un alto número de elementos apendiculares superiores, que se completa con la sección craneal en los ovicápridos, suidos y cérvidos, al contrario que los huesos axiales mal representados en todos los casos salvo los suidos.

En La Gavia III hemos observado que la sección craneal es la que mejor está representada en todos los taxones, y en el lado contrario, la peor documentada es la axial para équidos y ovicápridos, y los elementos apendiculares para bóvidos y suidos. En Humanejos se aprecia una tendencia similar al caso de La Gavia III, donde la sección craneal es la mejor conservada, salvo en el caso de los équidos, y los elementos con menor presencia corresponden a los axiales y apendiculares.

En La Cuesta se ha documentado el mismo patrón que en los otros casos. La novedad de este yacimiento es que en aquellos taxones con más restos se aprecia que cada taxón tiene un elemento predominante distinto: en bóvidos es la zona craneal, en équidos y ovicápridos los elementos apendiculares superiores, y en suidos el esqueleto axial y el craneal.

En Entreviñas I, al presentar una muestra tan escasa, sólo se han podido determinar estos parámetros en dos taxones, bóvidos y suidos. Por un lado, tenemos que la parte mejor documentada es la craneal en ambos animales $y$, para la zona con menor índice de representación, nos encontramos que para los bóvidos son los elementos apendiculares inferiores y para los suidos los elementos apendiculares superiores.

En Cerrocuquillo apreciamos que tanto bóvidos como équidos presentan un mismo patrón, donde el elemento craneal es el mejor representado y el esqueleto axial el menos numeroso. Para los ciervos el elemento con mayor predominio es la zona craneal y los elementos apendiculares superiores, mientras que en los suidos los elementos apendiculares superiores son los peor representados y los más importantes los elementos craneales.

\begin{tabular}{|c|c|c|c|c|c|c|c|c|c|c|c|c|}
\hline & \multicolumn{2}{|c|}{ La Guirnalda } & \multicolumn{2}{|c|}{ La Gavia III } & \multicolumn{2}{|c|}{ Humanejos } & \multicolumn{2}{|c|}{ La Cuesta } & \multicolumn{2}{|c|}{ Entreviñas I } & \multicolumn{2}{|c|}{ Cerrocuquillo } \\
\hline & Más & Menos & Más & Menos & Más & Menos & Más & Menos & Más & Menos & Más & Menos \\
\hline Bóvido & $\begin{array}{c}\text { Ap. } \\
\text { Superior }\end{array}$ & Axial & Craneal & $\begin{array}{c}\text { Ap. } \\
\text { Superior }\end{array}$ & Craneal & $\begin{array}{c}\text { Ap. } \\
\text { Inferior }\end{array}$ & Craneal & $\begin{array}{c}\text { Ap. } \\
\text { Inferior }\end{array}$ & Craneal & $\begin{array}{c}\text { Ap. } \\
\text { Inferior }\end{array}$ & Craneal & Axial \\
\hline Équido & $\begin{array}{c}\text { Ap. } \\
\text { Superior }\end{array}$ & Axial & Craneal & Axial & $\begin{array}{c}\text { Ap. } \\
\text { Superior }\end{array}$ & Axial & $\begin{array}{c}\text { Ap. } \\
\text { Superior }\end{array}$ & Axial & & & Craneal & Axial \\
\hline Ciervo & Craneal & Axial & Craneal & & Craneal & & & & & & $\begin{array}{c}\text { Craneal/Ap. } \\
\text { Superior }\end{array}$ & Axial \\
\hline Ovicáprido & $\begin{array}{c}\text { Craneal/ } \\
\text { Ap. } \\
\text { Superior }\end{array}$ & Axial & Craneal & Axial & Craneal & $\begin{array}{c}\text { Ap. } \\
\text { Inferior }\end{array}$ & $\begin{array}{c}\text { Ap. } \\
\text { Superior }\end{array}$ & $\begin{array}{c}\text { Ap. } \\
\text { Inferior }\end{array}$ & & & & \\
\hline Suido & Craneal & $\begin{array}{c}\text { Ap. } \\
\text { Inferior }\end{array}$ & Craneal & $\begin{array}{l}\text { Ap. Superior/ } \\
\text { Inferior }\end{array}$ & Craneal & $\begin{array}{c}\text { Ap. } \\
\text { Inferior }\end{array}$ & $\begin{array}{c}\text { Craneal/ } \\
\text { Axial }\end{array}$ & $\begin{array}{c}\text { Ap. } \\
\text { Inferior }\end{array}$ & Craneal & $\begin{array}{c}\text { Ap. } \\
\text { Superior }\end{array}$ & Craneal & $\begin{array}{c}\text { Ap. } \\
\text { Superior }\end{array}$ \\
\hline
\end{tabular}

Tabla 7. Representación de los yacimientos y los taxones más significativos en la muestra, en relación a las secciones anatómicas más o menos abundantes. 
Según se desprende de los datos mostrados en los perfiles esqueléticos de estos yacimientos en ambas etapas, se puede concluir que las muestras óseas se caracterizan por un gran sesgo osteológico. En la mayor parte de los yacimientos la sección craneal suele ser la predominante en contraposición a los elementos axiales, lo cual puede relacionarse con la mayor densidad de unos respecto a otros y sus mayores posibilidades de conservación. Por tanto, es posible que detrás de la representación esquelética diferencial de cada taxón haya razones tafonómicas que puedan explicarlas, por ejemplo la acción de perros que muerdan y alteren las muestras óseas, entre otras.

Por otra parte, y a pesar del sesgo osteológico de las muestras, la presencia de todas las partes anatómicas en los yacimientos con independencia de sus frecuencias sugieren que los animales generalmente llegaron completos a los yacimientos, pudiendo ser sacrificados en el mismo lugar o transportados completamente, salvo particularidades concretas en situaciones determinadas a las que no nos vamos a referir en este trabajo, como por ejemplo la selección de ciertas partes como las astas de ciervo para el procesado de materia prima o la deposición completa de animales enteros en ciertos silos o estructuras, cuyo significado escapa al enfoque de este estudio.

\section{Discusión y puesta en valor de los datos en su contexto}

Cuando aunamos los datos que hemos mostrado y los relacionamos con los existentes para la Primera Edad del Hierro de otros yacimientos como Ecce Homo (Morales 1980), Cerro San Antonio (Chaves et al. 1991), Puente Largo del Jarama (Liesau 1998a), Arroyo Culebro (Liesau 1998b y Orri y Nadal 2002) (no se toman los datos de la necrópolis de Arroyo $\mathrm{Cu}-$ lebro D), La Capellana (Liesau 1998a) y Las Camas (Yravedra 2007b); y la Segunda Edad del Hierro procedentes de El Cerrón (Miguel y Morales 1994), La Gavia (Urbina et al. 2005), Cerro Redondo (Miguel 1985) y Llano de la Horca (Cerdeño et al. 1992 y López y Morales 2012), así como los datos inéditos de las memorias arqueológicas de La Ribera (García y Vila 2003), Pista de Motos (Yravedra 2007c), Cerro de las Brujas (Rodríguez 2011), El Esparragal (Riquelme y Riquelme 2006) y El Malecón (Alarcón y Salso 2003), nos juntamos con un volumen de datos bastante importante. Sin embargo, y a pesar de lo que pudiera parecer, en realidad las informaciones zooarqueológicas disponibles son escasas en comparación al número de yacimientos de la Edad del Hierro excavados o conocidos para esta región en este periodo.

De este modo podemos decir que el volumen de datos faunísticos existente, en proporción al número de yacimientos conocidos para la Edad del Hierro en el centro de la Península Ibérica, es relativamente bajo y además tiene el problema de presentar una información zooarqueológica bastante heterogénea de unos sitios a otros (Yravedra y Estaca 2014). Así en muchos yacimientos se especifican informaciones como el tratamiento del NR, pero en la mayor parte de los casos otro tipo de informaciones como el MNI, los perfiles esqueléticos, los patrones de mortandad o los análisis tafonómicos brillan por su ausencia (Yravedra y Estaca 2014). Cuando hablamos del MNI el número de yacimientos desciende ya que Ecce Homo, Puente Largo del Jarama y La Capellana no presentan esta información. El número de sitios con análisis de los perfiles esqueléticos desciende drásticamente y los que tratan aspectos como la tafonomía se limitan al yacimiento de Las Camas (Yravedra 2007b).

Por tanto, unificando toda la información obtenida desde una perspectiva taxonómica apreciamos que el taxón predominante tanto en NR como en MNI, en toda la Edad del Hierro, son los ovicápridos, representados por ovejas y cabras. Si nos centramos en cada período vemos que para la Primera Edad del Hierro destacan los ovicápridos en el NR, salvo en el caso de Las Camas, seguido en importancia por la cabaña bovina y el cerdo a continuación. La cuarta especie más importante la constituyen los équidos, con frecuencias inferiores al $10 \%$ en casi todos los casos y, por último, el perro, que está muy mal representado.

Este mismo esquema se repite en el MNI, donde los ovicápridos están representando en torno al $40 \%$ de los individuos documentados en cada yacimiento, los bóvidos no alcanzan el $20 \%$ del MNI salvo en el caso de Las Camas que tiene un porcentaje mayor, los suidos no superan el $15 \%$ y los caballos se sitúan en porcentajes inferiores al 10\%.

En la Segunda Edad de Hierro se mantiene el predominio de la cabaña lanar tanto en NR como en MNI, seguido del ganado vacuno y porcino. Pero, con relación al primer momento 
de la Edad del Hierro, se producen ciertas variaciones en correspondencia con el $\mathrm{NR}$, donde se registra un aumento porcentual de los équidos, que duplican su valor -seguramente por la generalización del burro-, seguidos de cerdos y perros (Yravedra y Estaca 2014).

Es significativo como en esta Segunda Edad del Hierro parece ir aumentando la importancia de bovinos, equinos y suidos no sólo por su aumento porcentual, sino porque en algunos sitios como La Gavia III, el Cerro de las Brujas o La Guirnalda, algunos de estos taxones alcanzan frecuencias parecidas a las de los ovicápridos e incluso pueden llegar a desplazar a los ovicaprinos como especie principal, como por ejemplo en Entreviñas I o El Cerrón.

En el MNI se confirma lo mismo que en el NR, predominando casi siempre los ovicápridos que suelen representar el 35-50\% de los individuos. Los ovicápridos sólo superan el 50\% del MNI en el Cerro Redondo y el Llano de la Horca (López y Morales 2012). El aumento en importancia de otras cabañas ganaderas puede observarse en los bovinos que suelen suponer el $20 \%$ de los individuos, así como en los suidos que oscilan entre el $15-20 \%$ o los équidos con el $10-15 \%$.

Por tanto, se puede decir que hay una evolución en la que se pasa de una ganadería centrada en la cría de ganado lanar a otra en la que se vislumbra una diversificación hacia otros taxones, como los bóvidos y los suidos en relación con el NR. Esta diferencia es importante no sólo en términos del mayor contenido cárnico de estas especies, sino también de su movilidad, ya que se trata de animales que, para facilitar su engorde, suelen permanecer en un rango de espacio muy limitado, en ocasiones circunscripto a los alrededores del poblado, a menos que circunstancias apremiantes (v.gr. escasa cubierta vegetal) hagan imprescindible su movilización.

Tras la exposición de estos datos y en vista de la visión tradicional se podría pensar que la economía se basaba en la producción cárnica (Iborra 1999), pero, en contraposición, otros estudios realizados por Liesau y Blasco (1999), Collard et al. (2010) o Vidal y Maicas (2010), entre otros, demuestran que la utilización de la fauna con fines alimenticios no era ni el único propósito, ni el fin último, como lo ejemplifican los numerosos usos de la fauna que se han constatado desde la prehistoria hasta la actualidad.
Si nos centráramos sólo en términos alimenticios, pueden hacerse ciertas matizaciones, puesto que no es lo mismo el consumo de un individuo adulto que uno juvenil o infantil, debido a que el predominio de unos u otros puede tener diferentes connotaciones económicas y sociales. Si bien en los datos anteriormente expuestos, en ambos momentos cronológicos, los individuos adultos son los más abundantes, al analizar cada período por separado, se aprecia que para la Primera Edad del Hierro más del 30\% de los ovicápridos frecuentemente representan individuos infantiles y juveniles. Las vacas muestran porcentajes inferiores al $20 \%$ y, en el caso de los suidos, su representación puede llegar a superar el $40 \%$ de los individuos, como sudece en el caso de La Cuesta. Por tanto, con estos datos se puede plantear la hipótesis de que, para este primer momento, la ganadería buscaba no sólo la producción de carne, sino también la de otros productos (Sherratt 1981), como podrían ser la lana o la leche, como se comprueba en los estudios de manejo de rebaños (Cambero 1999).

Esta tendencia varía ligeramente en la Segunda Edad del Hierro donde, si bien la predominancia de los adultos se mantiene o aumenta en las cabañas ovina y bovina, las vacas no superan el 10\% entre las edades juveniles e infantiles, salvo en los asentamientos de La Cuesta y La Gavia III donde este porcentaje aumenta hasta el 30\% de los individuos juveniles. Y en los ovicápridos, por otro lado, la presencia de no adultos desciende al $20 \%$, y sólo en el caso de los suidos mantiene la tendencia descrita anteriormente. Por último, los équidos están muy poco representados, pero en ellos también destacan los adultos (Yravedra y Estaca 2014). Estos patrones podrían leerse como indicadores de que la funcionalidad de los animales se orienta más hacia la obtención de productos como la lana o la leche, como describiremos a continuación, o la fuerza de trabajo aportada por animales como el buey y el burro para las labores agrícolas (Urbina et al. 2005).

Aun así se ha apreciado el consumo de algunas crías indicando por tanto un pequeño exceso de producción de determinados animales o su sacrificio para obtener un mejor rendimiento lácteo de las madres. Un caso claro de este manejo podría ser el de Humanejos, que presenta gran número de neonatos de suidos, que por el ciclo vital de este animal podemos suponer que se consumieron tras su alumbra- 
miento sobre el mes de junio. No obstante, como hemos observado en la mayor parte de los individuos jóvenes, de la Primera y Segunda Edad del Hierro, su mortalidad coincide con los períodos de matanza en la época otoñal.

Llegados a este punto, hay que plantearse cómo se procesó esa carne para su consumo. Para ello contamos con los estudios sobre la representación anatómica, que si bien hasta el momento su análisis no ha sido muy frecuente entre los estudios zooarqueológicos, se puede decir que de forma global a partir de los datos mostrados en los yacimientos analizados, todos los taxones principales -ovicápridos, bóvidos, suidos y équidos- tienen huesos de todas las secciones anatómicas, aunque haya partes poco representadas como la sección axial. Esta presencia de huesos de todas las porciones anatómicas podría indicar que todos los taxones fueron aportados completos al yacimiento, teniendo su consumo lugar en el mismo asentamiento o en zonas muy próximas del mismo. La ausencia de las partes esqueléticas que hemos observado puede deberse al sesgo osteológico producido por procesos tafonómicos varios, como la fragmentación, as alteraciones diagenéticas y la acción de fenómenos predeposicionales como la acción de perros. De este modo es frecuente encontrar marcas de diente en los huesos producidas seguramente por los perros que vivieron en el yacimiento.

El análisis de trazas antrópico ha mostrado marcas de desollado, desarticulación, evisceración y descarnado sobre todos los taxones, incluidos animales minoritarios como los perros, que presentan huesos con marcas de corte en individuos adultos, infantiles y juveniles (Yravedra 2007a, b, c, d, 2009a, b y Estaca e Yravedra 2010, 2011a y b). Con relación al consumo de los perros, cabe destacar los análisis de las dos muestras tomadas para el estudio de sedimento contenido en algunos recipientes cerámicos del Llano de la Horca (Märtens et al. 2009:213):

1. Olla usada como probable basurero (dada la cantidad y variedad de fauna comestible con señales de descarnado y descuartizamiento) con restos de perro o lobo, oveja, conejo, cerdo, cáscara de huevo de especie indeterminada (aunque sabemos que había gallina en alguna ocupación), semillas carbonizadas, microfauna, etc.

2. Recipiente que contenía un cocido de carne de conejo y perro o lobo, con harinas de trigo, cebada y bellota.
Esto nos vuelve a llevar a la idea de que todas las especies una vez que cumplieron su función en vida, ya fuera por su lana, su leche, su fuerza de trabajo, etc., se procedía a su consumo cárnico.

Se ha podido advertir que las marcas de cortes, en la mayoría de los yacimientos en los que se mencionan este tipo de estudios, aparecen en las mismas partes anatómicas, sobre todo en las diáfisis de huesos largos. Así mismo se ha documentado que en animales de talla grande como el caballo o la vaca algunos huesos como el húmero o el fémur se fracturan para la obtención de la médula (Yravedra 2007b y 2012).

Por el contrario, en otros huesos con menos poder alimenticio este tipo de proceso de fracturación no se produce, como por ejemplo en los metapodios. De este modo un alto porcentaje tanto de metacarpos como de metatarsos fueron depositados completos, si bien algunos de ellos han llegado fracturados a nuestros días por procesos postdeposicionales diagenéticos en estado seco. En el caso de otros huesos como los radios y las tibias presentan un patrón diferente ya que aparecen indistintamente fracturados o no. En los animales de talla pequeña como ovicápridos y suidos se aprecia que los huesos no suelen estar fracturados para la obtención del tuétano; sus fracturas están más asociadas a alteraciones producidas por agentes más destructivos como el perro o el cerdo, o a fracturas en seco. Sólo se han documentado tajos en algunas pelvis, fémur, tibias, escápula y húmeros, que tienen más relación con el despiece/desarticulación que con la obtención de médula.

Así mismo, las marcas de evisceración y desarticulado están indicando el proceso por el cual estas poblaciones despiezaban no sólo para su consumo y cocción inmediata, tratamiento del que sí tenemos evidencias a través de los huesos deshidratados de color crema presentes en la muestra, sino para su conservación en un futuro a corto y medio plazo. En este último caso, cabe la posibilidad de que en esta época se emplearan métodos básicos de conservación, donde la carne se deshidrataría impidiendo la acción de los microorganismos que producen su descomposición, como lo menciona el autor romano Catón el Viejo en su obra De Re Rustica. Para ello la carne se secaría a través de la acción combinada del calor y el humo o del aire seco y frío, además de emplear productos antisépticos como los que 
desprende el fuego de leña en el ahumado o la sal de las salazones (Torres 2005: 51). Es importante destacar que la sal es fundamental no sólo para el tratamiento de la carne sino para la fabricación de quesos y el tratamiento de pieles (Valiente et al. 2014), que no se desperdiciaría en contextos como el que estamos tratando, pese a no tener evidencia física de ello, muy probablemente debido a su pobre conservación o a la falta de estudios sistemáticos que mediante análisis químicos de suelos detecten estas sustancias, pero no podemos desdeñar la posibilidad de que la sal se utilizara de manera recurrente debido a la presencia de salinas en el centro peninsular (Puche y Mazadiego 2000). Otro método de conservación de la carne empleado en la época sería posiblemente la conserva en grasa o manteca, que permite la preservación de la carne ya preparada. En la necrópolis de Las Ruedas en Pintia, por ejemplo, se han encontrado indicios de preparados de este tipo (Sanz et al. 2003: 152 y Juan y Matalama 2003: 314-316).

Tras analizar las evidencias dejadas por el consumo cárnico es importante destacar, en este punto del trabajo, las aportaciones que el animal tuvo en la realización de ciertas actividades artesanales, ya fuera con el animal vivo, como la producción textil que únicamente requiere de los animales la lana o el pelo, o la obtención de leche para procesar lácteos, o con el animal sacrificado, como el curtido de la piel, o el uso de los huesos, cuernos y astas utilizados en la industria ósea, etc.

Centrándonos en las actividades con el animal vivo, nos interesa un producto básico, la leche, que es la base de los productos secundarios. Su análisis no ha tenido una gran atención por parte de la investigación (Sherrat 1981), esto se debe, desde nuestro punto de vista como el de otros autores (Liesau y Blasco 1999; Torres y Sagardoy 2004; Torres 2007), a que la mayoría de los utensilios que se debieron de emplear para su procesado fueron artefactos orgánicos perecederos como maderas, cueros, etc. y que, por tanto, no han dejado huella en el registro arqueológico. De la misma manera, es bien sabida la dificultad de detectar la caseína incluso en aquellos recipientes que se sabe que estuvieron en contacto con la leche.
La leche de vaca, oveja y cabra debió procesarse de manera rápida debido a que es un producto delicado en su conservación, no así cuando los productos ya están manufacturados mediante su fermentación, dando lugar por un lado a los yogures, cuajadas, requesones, etc., con una durabilidad relativamente corta, $y$, por otro, a los quesos y mantequillas de larga duración que permiten su almacenaje y su transporte sin mayores problemas, por lo que eran dos productos muy útiles para tener una reserva alimenticia de proteínas sin tener que proceder a la matanza de los animales.

Por otra parte, la producción textil debió ser una de las actividades fundamentales en estas poblaciones, asociado según algunos autores a un trabajo específico de las mujeres (Torres 2005 y 2011; Bonet y Vives-Ferrándiz 2011). Los tejidos se realizaron por un lado, con fibras vegetales, de las cuales sería fundamental el lino, aunque también se habrían empleado fibras de algodón, como se ha documentado en el yacimiento de Cerrocuquillo en la Primera Edad del Hierro (Torija et al. 2010). Aun así seguramente la fibra más usada para la realización de tejidos sería la lana de ovejas adultas. Ello se podría sustentar con los patrones de mortandad que hemos obtenido, ya que en un alto porcentaje las ovejas sacrificadas eran adultas, indicando por tanto la importancia del animal en vida, probablemente para el aprovechamiento de la lana como materia prima.

Para llegar a obtener el producto final en forma de fibra, lo primero que se debía de realizar era el esquileo de las ovejas, siendo esta una actividad farragosa hasta la aparición de las tijeras en la Segunda Edad del Hierro como las documentadas en el Llano de la Horca (Ruiz Zapatero et al. 2012: 340) o en la Dehesa de la Oliva (Cuadrado 1991), que mejoraron sensiblemente las condiciones en que se llevaba a cabo esta actividad. Tras la obtención de la materia prima se procedería a su limpieza a través del rastrillado y el cardado. Es posible que en un primer momento se usasen cardadores naturales, como el cardo seco, para posteriormente pasar a la utilización del peine cardador de metal, como el encontrado en el Llano de la Horca (Ruiz Zapatero et al. 2012) (Fig. 4). 

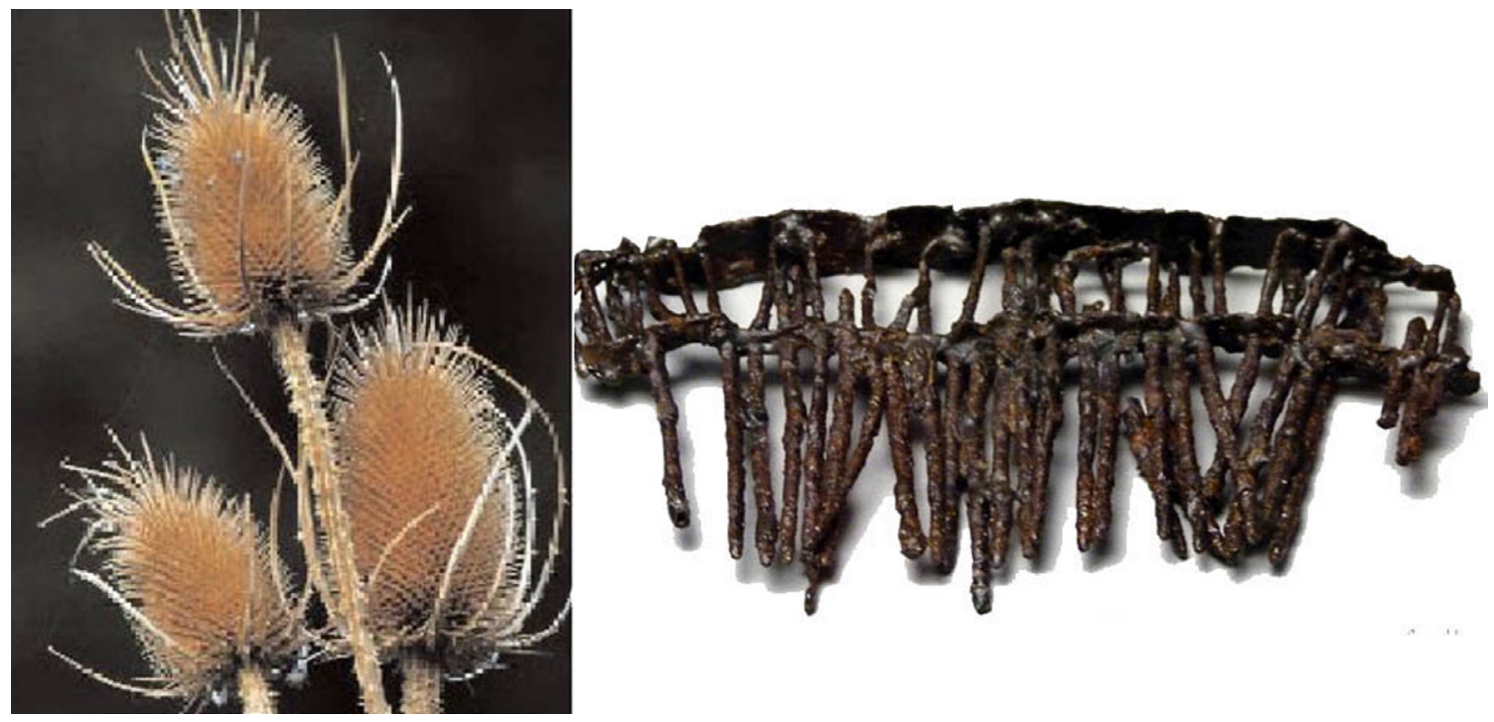

Figura 4. Cardadores de lana. A la izquierda un cardo seco, seguramente empleado en la Primera Edad del Hierro. A la derecha un cardador de metal de la Segunda Edad del Hierro perteneciente al Llano de la Horca.

Con ello se llegarían a realizar unas mechas que con la ayuda del huso se podían hilar. Este huso era un vástago ligero de madera, hueso, caña, etc., con una longitud aproximada de entre 20 y $30 \mathrm{~cm}$. El mejor ejemplo que se ha documentado hasta el momento es el vástago de huso hallado en Cerro Redondo, que se encontró con un trozo de hilo de lana atado a él (Blasco y Alonso 1985). En uno de los extremos del vástago se ponía una fusayola, normalmente realizada en cerámica, hueso o madera y con forma troncocónica o bitroncocónica. En nuestro caso, las fusayolas recogidas en los yacimientos analizados corresponden a la Segunda Edad del Hierro. Así mismo, son todas de cerámica con excepción de un ejemplar documentado en el asentamiento de La Guirnalda, que estaba realizada en hueso (Fig. 5). Con las fusayolas lo que se conseguía era favorecer la rotación y disminuir la oscilación en los giros rápidos que, unido al peso de las mismas, facilitando la obtención de las madejas y las dejaba listas para pasar al procesado en el telar (Castro 1980: 144).

Es habitual encontrar grupos de fusayolas en los ámbitos domésticos, donde debieron de situarse los telares. En nuestro caso, y siempre hablando de la Segunda Edad del Hierro, nos encontramos que estos elementos se localizan tanto en ámbitos domésticos, como su- cede en la zona del derrumbe de una cabaña en La Guirnalda, como repartidas por distintos ámbitos, como en el Sector II de La Gavia III. También se han localizado en áreas de producción artesanal, como sucede en el caso de La Cuesta, que se podría vincular con la fuerte concentración que se encontró en Fuente de la Mora (Leganés, Madrid), en la que se evidencia un área especializada de trabajo (Vega et al. 2009).
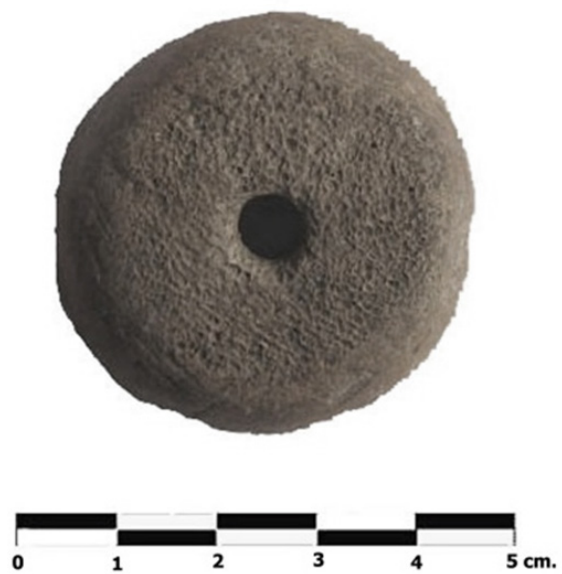

Figura 5. Fusayola realizada en hueso del yacimiento de La Guirnalda (Foto cedida por Jorge Morín, AUDEMA) 
Otro elemento a tener en cuenta son las pesas de telar o ponderas de formas troncocónicas y rectangulares, que servían para tensar los hilos en telares verticales que, al ser construidos en madera, no tienen fácil visualización arqueológica, salvo por los agujeros en el suelo para fijar los pies o por la concentración de las pesas de telar en determinadas zonas de habitación, como ocurre en las áreas domésticas en La Guirnalda, ámbito 1 (UE 25018), donde aparecen in situ (Agustí 2007) y en La Gavia III, Sector II, para el segundo período (Morín 2008a). Como vemos, la actividad textil y, por extensión, el aprovechamiento de la lana parece ser una actividad frecuente en el tratamiento de la fauna de los sitios estudiados.

Si la lana debió servir como principal materia prima para la vestimenta, no se puede despreciar el uso de la piel y el cuero de los diversos animales, ya fueran domésticos o salvajes, transformándolos y manufacturándolos. No sólo emplearían la piel con el pelo, sino que también lo curtirían empleando ambos elementos no sólo para la vestimenta y calzado, sino para la elaboración de mobiliario, recipientes, cinturones, corazas, cascos, arreos de caballos, etc. (Cuadrado 1991; Liesau y Blasco 1999; Torres 2005: 108).

Es importante tener en cuenta que tras el sacrificio de una res o la caza de un animal, tanto la carne como la piel deben procesarse rápidamente para evitar su descomposición y mantener sus condiciones de flexibilidad y resistencia. Por tanto, hay que desollar al animal, como se evidencia en todos los yacimientos aquí analizados. Las marcas de desollado son frecuentes, halladas por ejemplo en las falanges de vacas y caballos en La Guirnalda para la Primera Edad del Hierro, en Cerrocuquillo o en Las Camas.

Tras obtener la piel, esta debe limpiarse a través de raspadores y abrasivos como la ceniza para eliminar cualquier tipo de grasa o sebo. A continuación la piel podía introducirse en una disolución de tanino pudiendo estar en tratamiento hasta medio año (Torres 2005: 109). Tras su curtido se pasaba a su curación posiblemente a través de orines gracias a su gran contenido en ácido úrico, consiguiendo con la misma gran flexibilidad. Por último, se podría proceder a su decoración usando la sangre, ya fuese como colorante o aglutinante (Vidal y Maicas 2010).

Es importante no olvidarse ni de los tendones ni de las vísceras, de los cuales en nuestro registro óseo han quedado evidencias, por un lado, en las marcas de desarticulación, cuyo trazado permitiría la extracción de tendones particularmente en los huesos largos y, por otro lado, en las marcas de evisceración halladas en el interior de las costillas. Ejemplos de obtención de tendones encontramos en todos los yacimientos aquí tratados y para ambos momentos cronológicos, y podrían haber sido utilizados como un cordel resistente y flexible, empleándose en prendas de vestir, ligamentos de útiles, etc. Las marcas tendientes a la obtención de las vísceras también se han documentado en algunos yacimientos estudiados. Con la evisceración se recuperaba fundamentalmente el estómago y los intestinos -además de otras partes también consumibles como el hígado, los riñones, etc.-, que podrían haber sido usados en ocasiones como recipientes de líquidos o como contenedor de la carne obtenida en la matanza y que posteriormente se curaban o secaban (Stewart 1984).

Una mención aparte se merecen las herramientas óseas, que suelen considerarse la principal categoría de restos animales no comestibles. En este trabajo no nos hemos detenido en el análisis de este material, pero creemos necesario hacer algunas consideraciones. En los yacimientos estudiados la industria ósea es testimonial, recogiendo en la mayoría de los casos piezas apuntadas, biseladas y espátulas. Seguramente, como exponen Vidal y Maicas (2010), muchos de los huesos que fueron quebrados para la obtención del tuétano se pudieron haber usado posteriormente para la realización de objetos óseos, en especial en el caso de huesos grandes.

Por último, no se nos puede olvidar otro uso, el del hueso como combustible, ya que los sobrantes de huesos serían de utilidad para economizar el gasto de combustible vegetal de los hogares (Yravedra et al. 2005; Vidal y Estaca 2014). Sin embargo, este tipo de comportamiento no parece haberse registrado en ninguno de los yacimientos estudiados, ya que no han aparecido grandes concentraciones de hueso carbonizado o calcinado muy fragmentado. No obstante, este tipo de usos u otros, como el de la fragmentación del hueso para obtener grasa, no deben ser descartados aunque de momento no tengamos evidencias que permitan su demostración. Así mismo, también se pudo haber utilizado el estiércol como combustible, ya que quema lentamente y mantiene una temperatura constante, una clara ventaja en la pre- 
paración de alimentos que requieren un largo tiempo de cocción (Vidal y Maicas 2010).

\section{Conclusiones}

Se ha pretendido dar una visión de conjunto de la fauna no sólo desde un punto de vista zooarqueológico, sino de las implicaciones que tuvo dentro de la sociedad de la Edad del Hierro. Así, se ha podido determinar que el taxón predominante en términos de NR y MNI en la Edad del Hierro, es el ovicaprino, seguido en importancia de los bóvidos, suidos y équidos, en este orden, aunque el porcentaje de équidos aumenta en la Segunda Edad del Hierro, igualándose a la de los suidos.

Uno de los principales objetivos de nuestro trabajo era correlacionar el registro faunístico de los yacimientos con la cultura material existente en cada sitio. Efectivamente hemos visto que hay aspectos que muestran esta correlación, así hemos observado como el predominio de adultos suele estar asociado al aprovechamiento en vida de los animales para la manufactura de lácteos, lana, piel, y otros productos, los cuales nos han dejado evidencias materiales dentro del registro arqueológico en forma de cardadores, pesas de telar, fusayolas... Así mismo, la presencia de algunos individuos infantiles y juveniles, sobre todo para la Segunda Edad del Hierro, puede deberse a un exceso de producción de determinados animales o a su sacrificio para obtener un mejor rendimiento lácteo de las madres, sobre todo en el caso de los ovicápridos. Pero en ninguno de los yacimientos analizados y conocidos para el mundo carpetano se han localizado asentamientos con pautas especializadas en el sacrificio de individuos jóvenes, que sugerirían algún tipo de especialización relacionada con la explotación de recursos cárnicos. Más bien lo observado apunta a todo lo contrario, yacimientos que focalizan la atención en la producción de lana, leche, fuerza de trabajo y demás productos derivados de estos animales.
Como perspectivas de futuro queremos destacar que, además de las conclusiones que hemos presentado en este trabajo, hay aspectos que sería interesante comprobar y que por falta de evidencias no hemos podido afrontar. Por ejemplo, analizar cómo se diferencian las deposiciones óseas de los yacimientos asociados a áreas de ocupación, como poblados, respecto a otro tipo de yacimientos, como las necrópolis. Hasta el momento sólo el Arroyo Culebro ha ofrecido datos faunísticos entre los yacimientos asociados a necrópolis, lo cual deja un bagaje insuficiente para poder ser comparado con los otros tipos de yacimientos. Además de la realización de análisis de espacialidad dentro de cada yacimiento para determinar, por ejemplo, zonas de cercado de animales o las implicaciones simbólico-religiosas que pudo tener la fauna. Así, en algunos asentamientos como en Las Camas se han detectado deposiciones intencionales de una mandíbula de perro y de équido sobre las bases de los postes principales de la cabaña más importante del yacimiento (Yravedra 2007b).

En definitiva, este estudio ha ofrecido información sobre la economía ganadera, mostrando que cabañas ganaderas son las más importantes, qué usos tuvieron, y cómo contribuyeron a la economía de estas poblaciones de la Edad del Hierro. Sin embargo, hay otros aspectos que aún quedan por dilucidar y sólo futuros análisis que traten estas nuevas líneas de investigación permitirán obtener más respuestas a las cuestiones planteadas y a otras que estarán por venir.

\section{Agradecimientos}

Este trabajo no hubiera sido posible sin el respaldo de mi director de tesis, el Dr. Yravedra, así como de Jorge Morín, Raúl Flores, Primitivo Sanabria, Isabel Baquedano, Alicia Torija, Montserrat Cruz y Basilio Garrido, por confiar en mí y dejarme estudiar los materiales de los siete yacimientos que en este trabajo se presentan.

\section{Bibliografía}

Agustí, E. (2007): Memoria de intervención arqueológica en el yacimiento de La Guirnalda (Quer, Guadalajara). Tomo 1. Madrid, AUDEMA, S.L.

Agustí, E.; Urbina, D.; Morín, J.; Villaverde, R.; Martínez Navarro, E.; de Almeida, R.; López, F. y Benito, L. (2012): La Guirnalda: un yacimiento de la Edad del Hierro en la provincia de Guadalajara. En Morín, 
J. y Urbina, D. (eds.): El primer milenio a. C. en la Meseta Central. De la longhouse al oppidum. Vol. 2. Madrid, AUDEMA, S.L.: 181-191.

Alarcón, A. y Salso, E. (2003): Informe arqueofaunístico del yacimiento arqueológico de "El Malecón". En Rodríguez Cifuentes, M. (ed.): Memoria de resultados de la excavación arqueológica realizada en el yacimiento del Malecón, Planta separadora de hidrocarburos (Plan Barajas, Madrid). Informe Inédito presentado por Gestión Patrimonio Cultural, S.L., depositado en el MAR.

Azcárraga, S. (2015): El ocaso de un pueblo. La Carpetania centro-septentrional entre la Segunda Edad del Hierro y la época romana (siglos III a.C.-I d.C.): el valle bajo del Henares. Zona Arqueológica, 18. Alcalá de Henares, Museo Arqueológico Regional.

Baquedano, E. (ed.) (2014): Primer Simposio Sobre los Carpetanos, Arqueología e Historia de un pueblo de la Edad del Hierro. Zona Arqueológica, 17. Alcalá de Henares, Museo Arqueológico Regional.

Baquedano, I.; Torija, A. y Cruz, M. (2010): Algunos apuntes sobre las excavaciones en curso del yacimiento de Cerrocuquillo (Villaluenga de la Sagra - Toledo). Actas de las II Jornadas de Arqueología de Castilla-La Mancha. Toledo, Diputación de Toledo: 117-156.

Blasco, Ma .C. y Alonso, M.A. (1985): Cerro Redondo. Fuente el Saz del Jarama. Excavaciones Arqueológicas en España, 143. Madrid, Ministerio de Cultura.

Boessneck, J. (1969): Osteological Differences between Sheep (Ovis aries aries Linné) and Goats (Capra hircus hircus linné). En Brothwell, D. y Higgs, E. (eds.): Science in Archaeology. Londres, Thames y Hudson: 331-358.

Bonet, H. y Vives-Ferrándiz, J. (eds.) (2011): La Bastida de les Alcuses. 1928-2010. Valencia: Museu de Prehistòria de Valencia.

Brain, C.K. (1969): The contribution of Namib desert Hottentot to understanding of Australopithecus bone accumulations. Scientific Papers in Namibian desert Research Station, 32: 1-11.

Brown, W.A.B. y Chapman, N.G. (1991a): The dentition of red deer (Cervus elaphus): a scoring scheme to assess age from wear of the permanent molariform teeth. Journal of Zoological London, 224: 519-536.

Brown, W.A.B. y Chapman, N.G. (1991b): Age assessment of red deer (Cervus elaphus) from a scoring scheme based on radiographs of developing permanent molariform teeth. Journal of Zoological London, 225: 85-97.

Cambero, P. (1999): Cuaderno de la explotación del ovino. Servicio agrario de Caja Duero. Salamanca.

Castro, Z. (1980): Fusayolas ibéricas, antecedentes y empleo. En Cypsela Vol. 3. Gerona, Museu d'Arqueologia de Catalunya. Generalitat de Catalunya. Departament de Cultura: 127-146.

Cerdeño, Ma.L.; Martín, E.; Marcos, F. y Ortega, J. (1992): El yacimiento prerromano de Santorcaz (Madrid). Arqueología, Paleontología y Etnografía, 3: 131-170.

Chaves, P.; Morales, A.; Serrano, L. y Torre, M.A. (1991): Informe faunístico. En Blasco, Ma .C.; Alonso, M.A. y Lucas, $M^{a}$.R. (coords.): Excavaciones en el poblado de la Primera Edad del Hierro del Cerro de San Antonio. Arqueología, Paleontología y Etnografía, 2: 167-171.

Collard, D.; Morris, J.; Perego, E. y Tamorri, V. (eds.) (2010): Food and drink in Archaeology 3. University of Nottingham Postgraduate Conference 2009. Londres: Prospect Books.

Consuegra, S. y Díaz del Río, P. (2007): El yacimiento de La Albareja (Fuenlabrada, Madrid): un ejemplo de poblamiento disperso en la Edad del Hierro. En Davila, A.F. (ed.): Estudios sobre la Edad del Hierro en la Carpetania. Registro arqueológico, secuencia y territorio. Zona Arqueológica 10 (2). Alcalá de Henares, Museo Arqueológico Regional: 131-152.

Couturier, M.A.J. (1962): Détermination de l'âge du Bouquetin des Alpes a l'aide des dents et des cornes. Mammalia, 25 (4): 453-461.

Cuadrado, E. (1991): El castro de la Dehesa de la Oliva. Arqueología, Paleontología y Etnografia, 2: 191255.

Dávila, A. (ed.) (2007): Estudios sobre la Edad del Hierro en la Carpetania. Zona Arqueológica, 10. Alcalá de Henares, Museo Arqueológico Regional.

Estaca, V. e Yravedra, J. (2010): Informe Arqueozoológico del Yacimiento de Entreviñas I Fase I. Estudio realizado para Basilio Garrido. Manuscrito.

Estaca, V. e Yravedra, J. (2011a): Informe Arqueozoológico del Yacimiento de Entreviñas I Fase II. Estudio realizado para Basilio Garrido. Manuscrito.

Estaca, V. e Yravedra, J. (2011b): Informe Arqueozoológico del Yacimiento de La Gavia III. AUDEMA, S.A.. Manuscrito. 
Fernández, H. (2001): Ostéologie comparée des petites ruminants eurasiatiques sauvages et domestiques (genres Rupi Capra hircus, Ovis aries, Capra hircus et Capreolus): diagnose differentialle du squelette apendiculaire. Universidad de Génova, Facultad de Ciencias.

Flores, R. (2011): El yacimiento de Humanejos. En Blasco, C.; Liesau, C. y Ríos, P. (eds.): Yacimientos calcolíticos con campaniforme de la región de Madrid: Nuevos estudios. Patrimonio Arqueológico de Madrid, 6: 9-16.

Flores, R. y Sanabria, P.J. (2012): La Cuesta, Torrejón de Velasco (Madrid): Un hábitat singular en la I Edad del Hierro. En Morín, J. y Urbina, D. (eds.): El primer milenio a.C. En la meseta central de la longhouse al oppidum: 281-292.

Flores, R. y Sanabria, P.J. (2014): Actividades productivas en La Cuesta (Torrejón de Velasco, Madrid). Primer Simposio Sobre los Carpetanos, Arqueología e Historia de un pueblo de la Edad del Hierro. Zona Arqueológica, 17. Alcalá de Henares, Museo Arqueológico Regional: 203-209.

García, V. E. y Vila, S. (2003): Anexo 3: Estudio Faunístico del Yacimiento arqueológico: La Ribera. En Memoria de excavación del yacimiento de la Ribera. Obras de ampliación del aeropuerto de Madrid-Barajas, dentro de los proyectos constructivos "Galería de Balizamiento y Servicios", por debajo de la Pista de Vuelo 18L/36R y "Galería de Drenaje (Línea 7 a y 7 b)". Informe Inédito presentado por ARQUEO ESTUDIO, depositado en el MAR.

Garrido, B. (2012): Patrimonio, Identidad y Memoria de la Comarca de la Sagra: Propuesta museográfica para un futuro museo arqueológico en Illescas. Trabajo de Master universitario en educación y museos: Patrimonio, Identidad y Mediación cultural. Universidad de Alcalá de Henares. Manuscrito.

Guadelli, J.L. (1998): Détermination de l'age des caveaux fossiles et établissement des chasses d'age. Paléo, 10: 87-93.

Hilson, S. (1992): Mammal Bones and Teeth: An introductory guide to methods of identification. Londres, London Institute of Archaeology.

Iborra, M.P. (1999): Los recursos ganaderos en época ibérica: III Reunió sobre Economía en el Món Ibéric. Saguntum Extra 3.

Juan, J. y Matamala, J.C. (2003): Análisis de adobes, pigmentos, contenido de recipientes, instrumental textil, material lítico de molienda y cálculo dental humano procedente del yacimiento de Pintia. Apéndice I. En Pintia, un oppidum en los confines orientales de la región vaccea. Valladolid, Universidad de Valladolid: 311-323.

Lavocat, R. (1966): Faunes et Flores préhistoriques de L'Europe Occidentale. Collection L'homme et ses Origenes.

Liesau, C. (1998a): Análisis faunísticos de los yacimientos de «Huerta de los Cabreros», «Canera de la Flamenca» y «Puente Largo del Jarama» (Aranjuez, Madrid)". En Muñoz, K. (ed.): El poblamiento desde el Neolítico Final a la Primera Edad del Hierro en la cuenca media del Tajo: 617-646.

Liesau, C. (1998b): La fauna del Arroyo Culebro en el marco de la Edad del Hierro en la región de Madrid. Cuadernos de Prehistoria y Arqueología de la Universidad Autónoma de Madrid, 25 (1): 283-294.

Liesau, C. y Blasco, C. (1999): “Ganadería y aprovechamiento animal”. En Burillo F. (coord.): IV Simposio sobre celtiberos. Zaragoza, Universidad de Zaragoza.: 119-147.

López, Ma.D. y Morales, A. (2012): Entorno y actividades económicas. En Ruiz Zapatero, G.; Märtens, G.; Contreras, M. y Baquedano, E. (eds.): Los últimos carpetanos: el "oppidum" de El Llano de la Horca (Santorcaz, Madrid). Catálogo de la exposición celebrada del 18 de abril al 25 de noviembre de 2012. Alcalá de Henares, Museo Arqueológico Regional.

Mariezkurrena, K. (1983): Contribución al conocimiento del desarrollo de la dentición y el desarrollo del esqueleto postcraneal de Cervus elaphus. Munibe, 35: 149-202.

Märtens, G., Contreras, M., Ruiz, G. y Baquedano, E. (2009): El Llano de la Horca (Santorcaz). Un asentamiento carpetano en los albores de la romanización. Actas de las Terceras Jornadas de Patrimonio Arqueológico de la Comunidad de Madrid: 210-222.

Martín R. y Blázquez M.J. (1983): Apuntes de Osteología Diferencial en Mamíferos. Cátedra de Anatomía y Embriología. Facultad de Veterinaria. Universidad Complutense Madrid. Manuscrito.

Miguel, J. de (1985): "Informe sobre los restos faunísticos recuperados en el yacimiento de «Fuente el Saz» (Madrid)". En Blasco, M.C. y Alonso, M.A. (eds.): “Cerro Redondo. Fuente el Saz del Jarama”. Excavaciones Arqueológicas en España, 143. Madrid, Ministerio de Cultura: 301-350.

Miguel, J. de y Morales, A. (1994): "Informe sobre los restos faunísticos recuperados en el yacimiento de «El Cerrón», Illescas (Toledo)". En Valiente, S. (ed.): Excavaciones arqueológicas en "El Cerrón” de 
Illescas (Toledo): 206-211. Toledo, Servicio de Publicaciones de la Junta de Comunidades de Castilla-La Mancha.

Morales, A. (1980): Estudio de los restos óseos. En Almagro M. y Fernández Galiano D. (ed.): Excavaciones en el Cerro Ecce Homo (Alcalá de Henares, Madrid). Madrid, Diputación Provincial de Madrid.

Morín, J. (2008a): Memoria de intervención arqueológica en el yacimiento de la Gavia III (Villa de Vallecas, Madrid). Tomo 1. Madrid, AUDEMA, S.A.

Morín, J. (2008b): Memoria de intervención arqueológica en área. Tomo I: Sector 2 (Edad del Hierro) y Sector 3 (Edad del Bronce). Plan parcial del sector 9 del PGOU de Torrejón de Velasco (Madrid). Madrid, AUDEMA, S.A.

Morín, J., Agustí, E., Escolá, M., Pérez-Juez, A. y Urbina, D. (com) (2005): El cerro de la Gavia. El Madrid que encontraron los romanos. Museo Arqueológico Regional, Museo de San Isidro, Madrid.

Morín, J. y Urbina, D. (eds.) (2012): El primer milenio a.C. en la Meseta Central. De la longhouse al oppidum. 2 vols. Madrid, Auditores de Energía y Medio Ambiente.

Orri, E y Nadal, J (2002): Estudio de los restos faunísticos recuperados en los yacimientos arqueológicos PP5 PAU Arroyo Culebro (Leganés, Madrid). Informe ARTRA S:L: Manuscrito.

Pales, L. y Lambert, C. (1971): Atlas ostéologique pour servir à la identification des mammifères du quaternaire. Centre National de la Recherche Scientific. París.

Payne, S. (1985): Morphological distinction between the mandibular teeth of young sheep, Ovis aries and goats, Capra hircus. Journal of Archaeological Science, 12: 139-147.

Payne, S. y Bull, G. (1988): Components of variation in measurements of pig bones and teeth and the use of measurements to distinguish wild from domestic pig remains. Archeozoologia. Actes $d u 5^{\circ}$ congres International de Archeozoologie de Bordeaux, II (1-2): 27-66.

Pérez Ripoll, M. (1988): Estudio de la secuencia del desgaste de los molares de Capra pyrenaica de los yacimientos prehistóricos. Archivo de Prehistoria levantina, 18: 83-128.

Prummel, W. (1988): Distinguishing features en postcranial skeletal elements of cattle, Bos primigenius f, taurus and red deer, Cervus elaphus en Schiften aus der Archaeologish-Zoologischen Arbeistgroupe. Schleswig-Kiel. Heft, 12: 5-52.

Prummel, W. y Frisch, H.J. (1986): A guide for the distinction of species, sex and body size in bones of sheep and goat. Journal of Archaeological Science, 13: 567-577.

Puche, O. y Mazadiego, L. E. (2000): Salinas históricas de la Comunidad de Madrid: Carcaballana. Arqueología e Industria, (2-3),1999/2000: 81-90.

Riquelme, J.A. y Riquelme, I (2006): Estudio de fauna del yacimiento El Esparragal. En Petri, A. y Mendez, J.C.: Memoria final de las excavaciones arqueológicas en área realizadas en el Yacimiento de El Esparragal (Pinto, Madrid). Informe Inédito presentado por ARGEA CONSTRUCTORES S.L., depositado en el MAR.

Rodríguez, M. (2011): Estudio Arqueofaunísitico. Memoria de resultados de la excavación arqueológica realizada en el yacimiento Cerro de las Brujas (Pinto, Madrid). Informe Inédito presentado por Gestión Patrimonio Cultural, S.L., depositado en el MAR.

Ruiz Zapatero, G.; Märtens, G.; Contreras, M. y Baquedano, E. (2012): Los últimos carpetanos: el "oppidum" de El Llano de la Horca (Santorcaz, Madrid). Catálogo de la exposición celebrada del 18 de abril al 25 de noviembre de 2012. Madrid, Museo Arqueológico Regional.

Sanz, C.; Velasco, J.; Centeno, I.; Juan, J. y Matamala, J.C. (2003): Escatología Vaccea: nuevos datos para su comprensión a través de la analítica de residuos. En Sanz, C.; Velasco, J. (eds.): Pintia, un Oppidum en los confines orientales de la región vaccea. Valladolid, Universidad de Valladolid: 145-171.

Sherrat, A. (1981): Ploug and pastoralism: aspects of the secondary products revolution. En Hodder, I.; Isaac, G. y Hammond, N. (eds.): Pattern of the Past. Studies in honour of David Clarke: 261-305. Cambridge, Cambridge University Press.

Schmid, E. (1972): Atlas of Animal Bones for Prehistorians, Archaeologist an Quaternary Geologist. Amsterdan: Elsevier Publishing Company.

Stewart, H. (1984): Cedar. Tree of life to the northwest coast Indias. Londres: Douglas and McIntyre y University of Washington Press.

Torija, A.; Baquedano, I. y Cruz, M. (2010): Inhumaciones infantiles en el centro peninsular durante la protohistoria. Algunas novedades en el yacimiento de Cerrocuquillo. En Burillo, F. (ed.): VI Simposio sobre Celtiberos: Ritos y Mitos. Zaragoza, Fundación Segeda-Centro Celtibérico: 433-444. 
Torres, J. de (2013): La tierra sin límites. Territorio, sociedad e identidades en el valle medio del Tajo (s. $I X-I$ a. C.). Zona arqueológica, 16. Alcalá de Henares, Museo Arqueológico Regional.

Torres, J.F. (2005): La economía de los celtas de la Hispania Atlántica. Vol. II. Economía, territorio y sociedad. Serie Keltia, 28. A Coruña, Editorial Toxosoutos.

Torres, J.F. (2007): De los días y los trabajos: El calendario anual de las sociedades célticas de la Península Ibérica. Pasado y presente de los estudios celtas. Fundación ortegalia. Instituto de estudios celtas, Noia: 305-347.

Torres, J.F. (2011): El Cantábrico en la Edad del Hierro. Biblioteca Archaeologica Hispana, 35. Madrid, Real Academia de la Historia.

Torres, J.F. y Sagardoy, T. (2004): Economía ganadera en la zona cantábrica en el final de la Edad del Hierro. Kobie (serie Anejos), 6 (1): 315-332.

Urbina, D.; Morín, J.; Escolá, M.; Agustí; E. y Yravedra, J. (2005): La vida cotidiana. En Morín, J.; Agustí, E.; Escolá, M.; Pérez-Juez, A. y Urbina, D. (comp.): El cerro de la Gavia. El Madrid que encontraron los romanos. Madrid, Museo Arqueológico Regional, Museo de San Isidro: 147-176.

Valiente, S.; López-Cidad, F.; Ramos Sánchez, F. y Ayarzagüena, M. (2014): Los grandes poblados carpetanos cercanos a fuentes salobres: Cerro de La Gavia, Titulcia, Oreja y Valdelascasas. Primer Simposio Sobre los Carpetanos, Arqueología e Historia de un pueblo de la Edad del Hierro. Zona Arqueológica, 17. Alcalá de Henares, Museo Arqueológico Regional: 213-222.

Vega, J.J., Martín, P. y Pérez, D. (2009): El poblado de la Segunda Edad del Hierro del Cerro de La Fuente de la Mora (Leganés, Madrid). Actas de las Terceras Jornadas de Patrimonio Arqueológico de la Comunidad de Madrid. Madrid: 281-290.

Vidal, A. y Estaca, V. (2014): Burning Bones along Pre and Protohistory. Póster presentado en el XVII World Congress of the Prehistoric and Protohistoric Society 2014. Burgos.

Vidal, A. y Maicas, R. (2010): El pastor y su rebaño: usos no alimenticios de la fauna en las sociedades campesinas de la Península Ibérica. Relaciones de la Sociedad Argentina de Antropología XXXV: 257-278.

Yravedra, J. (2006): Tafonomía aplicada a zooarqueología. Madrid, Aula Abierta, UNED.

Yravedra, J. (2007a): Informe Arqueozoológico del yacimiento La Guirnalda, Quer (Guadalajara). AUDEMA, S.A. Manuscrito.

Yravedra, J. (2007b): Macromamíferos del yacimiento de la primera edad del hierro de las Camas Villaverde, Madrid)". En Morín, J. (ed.): Primer Simposio de la Investigación y Difusión Arqueopaleontológica en el Marco de la Iniciativa Privada. Madrid, AUDEMA, S.A.: 413-427.

Yravedra, J. (2007c): Informe Zooarqueológico del yacimiento Prehistórico de Pista de Motos (Villaverde, Madrid). Informe presentado por AREA Soc. Corp., depositado en el MAR. Manuscrito

Yravedra, J. (2007d): Informe Arqueozoológico de los niveles de la Edad del Hierro del yacimiento de Torrejón de Velasco (Madrid). Madrid, AUDEMA, S.A. Manuscrito.

Yravedra, J. (2009a): Zooarqueología y tafonomía en los comienzos de la Edad del Hierro. El yacimiento de Las Camas (Villaverde, Madrid). MArq Audema. Serie Zooarqueología 1.

Yravedra, J. (2009b): Informe Arqueozoológico del yacimiento La Cuesta (Torrejón de Velasco, Madrid). Pagadel, S.A. Manuscrito.

Yravedra, J. (2012): Zooarqueología, la fauna en la Primera Edad del Hierro. En Morín, J. y Urbina, D. (eds.): El primer milenio a.C. En la meseta central de la longhouse al oppidum. Madrid, AUDEMA, S.A.: 281-292.

Yravedra, J.; Baena J.; Arrizabalaga A. e Iriarte, M. (2005): El empleo de material óseo como combustible durante el Paleolítico Medio y Superior en el Cantábrico. Observaciones experimentales. Museo de Altamira. Monografias 20. Santander: 369-383.

Yravedra, J. y Estaca, V. (2014): Implicaciones de la zooarqueología sobre la economía en la Edad del Hierro. Primer Simposio Sobre los Carpetanos, Arqueología e Historia de un pueblo de la Edad del Hierro. Zona Arqueológica, 17. Alcalá de Henares, Museo Arqueológico Regional: 363-375. 